\title{
Early-term results of early coronary artery bypass graft surgery in patients undergoing primary percutaneous coronary intervention due to acute coronary syndrome
}

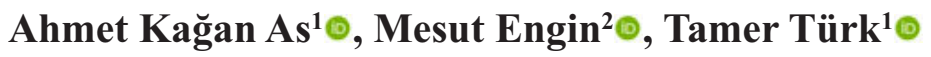 \\ ${ }^{1}$ Department of Cardiovascular Surgery, University of Health Sciences, Bursa Yüksek Ihtisas Training and Research Hospital, Bursa, \\ Turkey \\ ${ }^{2}$ Department of Cardiovascular Surgery, University of Health Sciences, Mehmet Akif Inan Training and Research Hospital, Şanlurfa, \\ Turkey
}

\section{ABSTRACT}

Objectives: This study aimed to investigate the early results of the patients presented with acute coronary syndrome (ACS) who underwent coronary artery bypass grafting (CABG) after percutaneous coronary intervention (PCI) to the culprit lesion.

Methods: Patients who underwent CABG between January 2011 and January 2014 were enrolled and divided into two groups. Group 1 (102 patients) was consist of the patients who were hospitalized with ACS and underwent CABG after a previous PCI. Group 2 (107 patients) was consisting of the patients who underwent elective $\mathrm{CABG}$ operation after elective coronary angiography.

Results: There was no statistically significant difference between the groups in terms of demographic features and preoperative risk factors. Preoperative use of angiotensin-converting enzyme inhibitor and levosimendan were significantly higher in group 1 compared to group 2. $(95(93.1 \%)$ vs. $89(83.1 \%), p=0.027)$. The operative variables were similar between two groups whereas the postoperative blood drainage amounts were significantly higher in group 1 than group $2(546.3 \pm 172 \mathrm{cc}$ vs. $424.2 \pm 185 \mathrm{cc}$, respectively, $p<0.001)$. The blood product usage was significantly higher in group 1 than in group 2 (3.3 \pm 1.8 units vs.1.7 \pm 0.9 units, respectively, $p<0.001$ ).

Conclusions: Early CABG operation after ACS is a safely applicable process with acceptable mortality and complication rates.

Keywords: Early CABG operation after ACS is a safely applicable process with acceptable mortality and complication rates.

C ardiovascular Diseases (CVDs) are the most common cause of mortality in developed countries as it is the expected case to be valid in developing countries in the future [1]. Coronary artery disease (CAD) is the most common form of cardiovascular diseases which is associated with high mortality and morbidity all over the world. Ischemic heart disease may clinically manifests as silent ischemia, stable angina pectoris, unstable angina pectoris, myocardial infarction (MI), heart failure, or sudden cardiac death. Acute coronary syndrome (ACS) is the name of a group of diseases that show different clinical manifes- 
tations in the course of coronary artery disease but entirely have a common pathophysiological mechanism [2]. The primary goal of the treatment in this clinical presentation, which manifests in a broad clinical spectrum ranging from unstable angina to ST elevation myocardial infarction (MI), is to eliminate acute obstruction in the coronary circulation by thrombolytic therapy, emergent percutaneous coronary intervention (PCI) or coronary artery bypass grafting (CABG) surgery. In this study, the patients presented with ACS who underwent PCI for the culprit lesion were examined. The patients who were scheduled to undergo CABG for coronary artery stenosis after PCI were compared with those who were scheduled to undergo elective CABG only. The aim of this study was to compare early (30 days) postoperative outcomes including mortality, morbidity and relevant factors between the two groups.

\section{METHODS}

\section{Patients}

This study was approved by the Medical Research Ethics Committee of our hospital. The data of 209 patients who were included in the study between January 2011 and January 2014 in our cardiovascular surgery department were retrospectively analyzed. According to the diagnostic and treatment protocol for ACS in our hospital, Coronary angiography (CAG) is scheduled within the first six hours after the onset of chest pain in patients presented with ACS. In our study, PCI was performed for the responsible coronary lesions detected by $\mathrm{CAG}$, and then the patients underwent CABG surgery because of extensive coronary lesions other than the culprit lesion. The patients who were scheduled to undergo CABG-only in the early period were also enrolled. In the same period, the patients who were scheduled to undergo elective CABG-only after elective CAG were also reviewed. Another patient group was randomly selected from the patients who had undergone CABG and included in the study as the control group. The 30-day postoperative mortality and morbidity data and the factors related to morbidity and mortality were investigated using the digital medical files of the patients. In this study, a total of 102 patients presented with ACS and had undergone CABG after PCI were classified as Group 1; in the same period, 107 patients who underwent elective CABG-only after elective CAG were classified as Group 2. The demographic characteristics, past medical history, perioperative data, and early postoperative mortality and morbidity outcomes were examined. The left ventricular function of all patients was evaluated by transthoracic echocardiography on the day before the surgery. As a clinical protocol, 87 (85.3\%) patients underwent $\mathrm{CABG}$ when preoperative troponin I level was below $3 \mathrm{ng} / \mathrm{ml}$ after MI. In this group, although they were hemodynamically stable, 15 (14.7\%) patients underwent $\mathrm{CABG}$ within the first three days due to a critical coronary artery lesion [severe left main coronary artery stenosis or severe proximal left anterior descending artery (LAD) stenosis] and ongoing angina despite optimal medical treatment after MI.After the first antiaggregant treatment given after PCI procedure, the antiaggregant treatment was terminated. If the stent was placed on the culprit lesion, clopidegrol $(75 \mathrm{mg} 1 \times 1)$ was started on the second postoperative day. Patients with a history of previous CABG operation, those who required additional surgery other than $\mathrm{CABG}$, and who needed emergency surgery due to hemodynamic instability were excluded from the study.

\section{Statistical Analysis}

SPSS 16.0 program was used for the analysis of the data. Categorical variables were expressed as number (\%) and continuous variables were expressed as the mean \pm standard deviation. The Fisher's Exact Test, Pearson's Chi-Square test, independent-samples t-test, and Mann-Whitney $U$ test were used for the relevant analyses. A $p$ value of $<0.05$ was considered as statistically significant.

\section{RESULTS}

In Group 1, there were a total of 102 patients (71 men and 31 women) with a mean age of $60.2 \pm 9.0$ years. In Group 2, there were a total of 107 patients (77 men and 30 women) with a mean age of $61.2 \pm$ 10.6 years. The preoperative variables of the patients in the two groups are shown in Table 1. The mean waiting time for the surgery was $9.7 \pm 4.6$ days in the patients who were admitted with ACS. All patients had undergone CABG between 1 to 21 days after hospi- 
Table 1. Preoperative characteristics of thepatients

\begin{tabular}{lccc}
\hline & $\begin{array}{c}\text { Group 1 } \\
(\mathbf{n = 1 0 2})\end{array}$ & $\begin{array}{c}\text { Group 2 } \\
(\mathbf{n = 1 0 7})\end{array}$ & $p$ value \\
\hline Age (years) & $60.2 \pm 9.0$ & $61.2 \pm 9.0$ & 0.489 \\
\hline DM & $49(48 \%)$ & $41(38.3 \%)$ & 0.156 \\
\hline HT & $84(82.3 \%)$ & $82(76.6 \%)$ & 0.307 \\
\hline HL & $68(66.6 \%)$ & $66(61.6 \%)$ & 0.453 \\
\hline Smoking & $74(72.5 \%)$ & $74(69.1 \%)$ & 0.590 \\
COPD & $26(25.4 \%)$ & $21(19.6 \%)$ & 0.310 \\
\hline Beta blocker use & $93(91.1 \%)$ & $88(82.2 \%)$ & 0.058 \\
\hline ACEI use & $95(93.1 \%)$ & $89(83.1 \%)$ & $\mathbf{0 . 0 2 7}$ \\
\hline Levosimendan use & $21(20.5 \%)$ & $11(10.2 \%)$ & $\mathbf{0 . 0 3 9}$ \\
\hline EF (\%) & $44.7 \pm 9.7$ & $46.5 \pm 9.3$ & 0.169 \\
\hline D & &
\end{tabular}

Data are shown as mean \pm standard deviation or $\mathrm{n}(\%)$. ACEI $=$ Angiotensin-converting enzyme inhibitor, $\mathrm{DM}=$ Diabetes Mellitus, HT = Hypertension, HL = Hyperlipidemia, COPD = Chronic Obstructive Pulmonary Disease, $\mathrm{EF}=$ Ejection Fraction

talization. There was no statistically significant difference between the two groups in terms of DM, HT, HL, smoking, and chronic obstructive pulmonary disease. The percentage of the patients using oral ACE inhibitors preoperatively was quite close in the two groups, but the difference was statistically significant [95 (93.1\%) vs. 89 (83.1\%), respectively; $p=0.027$ ]. The number of patients taking intravenous levosimendan in the preoperative period was higher in Group 1 than in Group 2 [21 (20.5\%) vs. $11(10.2 \%)$, respectively; $p=0.039$ ].

The variables that were evaluated during the surgery in the two groups are shown in Table 2 . The variables such as the number of grafts used in CABG, cross-clamping time, perfusion time, and LIMA usage among the perioperative data were compared between the groups. The LIMA graft was frequently used in both groups and there was no statistically significant difference between the two groups regarding LIMA use [97 (95\%) and 97 (90.6\%), respectively; $p=$ $0.213]$. There was no statistically significant difference between the two groups in terms of cross-clamping times $[67.1 \pm 27.2 \mathrm{~min}$ vs. $69.6 \pm 27 \mathrm{~min}$, respectively; $p=0.498$, perfusion times [91.8 \pm 36 min vs. $90.8 \pm$ 33.4 min, respectively; $p=0.843$ ] and the number of grafts used in CABG [3.3 \pm 1 vs. $3.3 \pm 0.9$, respectively; $p=0.901]$.

The patients were monitored in terms of early surgical complications and certain parameters in the intensive care unit until the discharge. The amount of drainage in the first 24 hours postoperatively was found to be $546.1 \pm 173 \mathrm{cc}$ in Group 1 and $424.2 \pm$ $185.6 \mathrm{cc}$ in Group $2(p<0.001)$. Parallel to this, the amount of blood products used in the first 48 hours postoperatively was $3.3 \pm 1.8$ units in Group 1 and 1.7 \pm 0.9 units in Group $2(p<0.001)$. The number of pa-

Table 2. Peroperativedatas of the patients

\begin{tabular}{lccc}
\hline & $\begin{array}{c}\text { Group 1 } \\
(\mathbf{n = 1 0 2})\end{array}$ & $\begin{array}{c}\text { Group 2 } \\
(\mathbf{n = 1 0 7})\end{array}$ & $\boldsymbol{p}$ value \\
\hline Number of the grafts & $3.3 \pm 1$ & $3.3 \pm 0.9$ & 0.901 \\
Cross-clamp time (min) & $67.1 \pm 27.2$ & $69.6 \pm 27$ & 0.498 \\
Perfusion time (min) & $91.8 \pm 36$ & $90.8 \pm 33.4$ & 0.843 \\
\hline LIMA use & $97(95 \%)$ & $97(90.6 \%)$ & 0.213 \\
\hline
\end{tabular}

Data are shown as mean \pm standard deviation or $\mathrm{n}(\%)$. LIMA $=$ Left internal mammary artery 
Table 3. Postoperative datas of the patients

\begin{tabular}{lccc}
\hline & $\begin{array}{c}\text { Group 1 } \\
(\mathbf{n}=\mathbf{1 0 2})\end{array}$ & $\begin{array}{c}\text { Group 2 } \\
(\mathbf{n}=\mathbf{1 0 7})\end{array}$ & $\boldsymbol{p}$ value \\
\hline Time to extubation (hours) & $12.6 \pm 6$ & $12.5 \pm 12.5$ & 0.932 \\
Drainage (cc) & $546.3 \pm 172$ & $424.2 \pm 185$ & $<\mathbf{0 . 0 0 1}$ \\
\hline Blood transfusion (unit:300 cc) & $3.3 \pm 1.8$ & $1.7 \pm 0.9$ & $<\mathbf{0 . 0 0 1}$ \\
\hline IABP use & $22(21.5 \%)$ & $13(12.1 \%)$ & 0.068 \\
\hline Pacemaker use & $7(6.8 \%)$ & $4(3.7 \%)$ & 0.312 \\
Atrial fibrillation & $27(26.4 \%)$ & $19(17.7 \%)$ & 0.129 \\
Infection & $17(16.6 \%)$ & $11(10.2 \%)$ & 0.175 \\
ICU stay (days) & $3.3 \pm 2.4$ & $2.4 \pm 1.4$ & $\mathbf{0 . 0 0 2}$ \\
\hline Hospital mortality & $5(4.9 \%)$ & $4(3.7 \%)$ & 0.470 \\
\hline $\begin{array}{l}\text { Data are shown as mean } \pm \text { standard deviation or n }(\%) . I C U=\text { Intensive care unit, IABP }=\text { Intraaortic balloon } \\
\text { pump }\end{array}$ & & &
\end{tabular}

tients requiring intra-aortic balloon pump (IABP) support in the postoperative period was higher in Group 1 than in Group 2, but the difference was not statistically significant between the two groups [22 (21.5\%) vs. $13(12.1 \%)$, respectively; $p=0.068]$. IABP support was used in the management of pump failure following cardiopulmonary bypass, and low cardiac output syndrome in both groups (Table 3 ).

In the overall study population, mortality was observed in $9(4.3 \%)$ patients. Three patients died due to low cardiac output and one patient died due to multiorgan failure in Group 1; while two patients died due to multi-organ failure and 3 patients died due to sepsis in Group 2. There was no significant difference between the two groups in terms of in-hospital mortality rate [5 (4.9\%) and $4(3.7 \%)$, respectively; $p=0.470]$.

\section{DISCUSSION}

In this study, we demonstrated that $\mathrm{CABG}$ can be performed in the early period with acceptable morbidity and mortality rates after PCI applied to the responsible coronary lesion in patients presented with ACS.

The acute coronary syndrome is the acute deterioration of underlying coronary artery disease by atherothrombotic occlusion of epicardial coronary arteries which leads to significant rates of mortality and morbidity. Successful interventional treatments have been developed over time for this acute devastating disease and significant improvements in in-hospital and long-term mortality rates have been achieved [25]. When ACS is diagnosed, treatment modality should be determined and the appropriate process for the required revascularization method should be initiated. Considering the coronary anatomy, the general condition of the patient and the interventional capability of the center, PCI or CABG should be decided for revascularization of the responsible coronary lesion or lesions. Recently, PCI has become the first choice treatment in the setting of ACS which results in satisfactory outcomes [5]. By the introduction of new devices and technical improvement in PCI procedures have allowed satisfying revascularization of lesions in epicardial coronary arteries and have significantly increased the availability of the procedure even in complex lesions. However, PCI can be inadequate to open target vessels in some cases such as left main coronary artery diseaseand multi-vessel disease or depending on the skill and experience of the cardiologist who performs the procedure. Under these circumstances, CABG becomes the preferred revascularization method. Along with the developments in invasive cardiology, it is possible to determine the limits of operability criteria, which is an important problem in cardiac diseases. In the previous studies till today, the precise indications for PCI and CABG have been determined. However, there are still debates on some lesions and patient characteristics. "Intervention-free life expectancy" and "event-free life expectancy" rather than the survival rates of patients after the procedure have become essential in determining indications [6]. 
Although both procedures can be performed in ACS, the choice and timing of the revascularization method are still controversial. In randomized studies, it has been emphasized that immediately identifying the patients who will benefit from early surgical revascularization is important [7]. According to coronary lesions viewed by $\mathrm{CAG}$, medical treatment of the patients is initiated after the decision for the choice of revascularization method (PCI or CABG). Five large randomized studies (RITA, GABI, CABRI, EAST, and BARI) have compared outcomes of the patients with multivessel disease after PCI and CABG. These studies concluded almost the same results. Both revascularization methods have similar risks of death and nonfatal myocardial infarction. However, patients treated with $\mathrm{CABG}$ were less likely to suffer from angina and to take anti-anginal drugs in the postoperative period. Further revascularization intervention was needed more frequently in patients who were treated with PCI. Moreover, it has been clearly shown in the BARI study that surgical method was superior to PCI in the management of diabetic patients with multi-vessel CAD.

We designed our study for the patients who were presented with ACS and underwent PCI for the culprit coronary lesion and then underwent elective isolated CABG for the other lesions in the early postoperative period. As a control group, we randomly selected the patients with stable CAD who underwent elective isolated $\mathrm{CABG}$ after elective $\mathrm{CAG}$ in the same time period in our hospital. Hemodynamically unstable patients were not included in our study. Coronary artery by-pass grafting was performed on an average of $9.7 \pm 4.6$ days in ACS patients. There was no significant difference between the two groups in terms of mortality. In a study including 12,227 patients between 2000 and 2012, Potluri et al. [7] have shown that there was a significant reduction in all-cause mortality in patients who underwent primary angioplasty after ACS and then who underwent CABG due to appropriate indications. The mortality rate of this study is very close to our mortality outcome. The general decline in mortality rate was emphasized overall ACS patients who had undergone CABG thereafter, especially in the group underwent CABG after PCI.

In our study, there was no statistically significant difference between the two groups in terms of the preoperative variables, demographic characteristics and concomitant diseases. Among the preoperative vari- ables, there was a statistically significant between the two groups regarding the medical treatments used in the preoperative period. There was a statistically significant difference between the two groups in terms of the use of oral ACE inhibitors and the use of intravenous levosimendan before the surgery. The use of oral ACE inhibitor was higher in Group 1. ACE inhibitors have the proven benefits such as increasing survival in ACS patients, reducing ventricular remodeling, having anti-atherogenic effect, and reducing the risk of death due to CAD [8-14]. In our study, we think that the use of oral ACE inhibitor was higher in Group 1 because of these reasons. In our hospital, the patients presented with ACS are treated with oral beta-blockers and oral ACE inhibitors routinely if there is no contraindication. Both drugs have been proven in terms of efficacy, so included in our routine clinical practice $[15,16]$. The treatment protocol of our center can explain the difference between the study groups regarding medications used.

Similarly, another preoperative medication that was statistically significantly different between the two study groups was the percentage of patients received intravenous levosimendan. Myocardial dysfunction develops frequently in acute and subacute periods after ACS. Early revascularization results in early reperfusion in infarct- or ischemia-related artery and a rapid improvement in ventricular function usually occurs after thrombolysis. However, if transmural injury and/or microvascular obstruction occurs, especially in the anterior wall after NSTEMI or STEMI, the pump failure accompanied with pathologic remodeling and clinical signs and symptoms of heart failure may complicate the acute phase and can result in chronic heart failure. Before $\mathrm{CABG}$, especially in the period after ACS, the situations such as the development of left ventricular dysfunction in echocardiography may be encountered which represent increased risk of morbidity and mortality after CPB. In these cases, preoperative levosimendan infusion would be beneficial in preventing increased mortality due to left ventricular dysfunction during and after cardiac surgery [17-19]. The fact that this treatment protocol, which was used significantly higher in Group 1, was applied for the medical treatment of the left ventricular dysfunction occurring after ACS would also explain the statistically significant difference between the two groups. In our postoperative data, although it is not 
statistically significant, greater use of IABP in Group 1 may explain that the increase in preoperative use of levosimendan was related to the intention to ameliorate the left ventricular dysfunction.

In the postoperative period, there was no statistically significant difference between the two groups in terms of the time spent on mechanical ventilation, the development of heart rhythm problems, infections, and the length of stay in the intensive care unit. However, in the postoperative period, there was a statistically significant difference between the two groups in terms of the amount of blood in drainage systems and the amount of blood and blood products used. Although the increased drainage in Group 1 did not require re-exploration for surgical revision of the bleeding, it became important with the increased need for blood and blood products. In addition to the mechanical approach to vascular pathology in the treatment of ACS, there are also medical treatment options to increase and maintain the blood flow and prevent re-stenosis after revascularization. Various drug groups such as thrombolytics, antithrombotics and anticoagulants have taken place in the treatment. These treatments, which are part of the treatment of ACS, bring the risk of bleeding problems together [20]. In the ACUITY study by Ben-Gal et al., after CABG was performed in patients with ACS and stable angina pectoris, the patients' mid- and long-term results have been examined. In this study, the authors mentioned that bleeding problems were experienced in the ACS group in the postoperative period due to antithrombotic and anticoagulant therapy used in the treatment of 1ACS preoperatively [21]. In our study, we obtained similar results and there was no reoperation need for bleeding revision, which suggest that this treatment protocol is suitable and its implementation prior to $\mathrm{CABG}$ does not create essential problems in the postoperative period.

\section{Limitations}

This study is a retrospective study carried out in two homogeneous groups of patients. Our results may have been affected by our treatment methods. Relatively small number of patients is also a limitation of the study. The surgical interventions had been performed by different surgeons which. make the standardization difficult. Our patients were analyzed for in-hospital mortality and morbidity. For this reason, its mid-term and long-term results could not be assessed. In addition, patients with hemodynamic instability after myocardial infarction were excluded. The patients were operated at least 24 hours after myocardial infarction. Therefore, the data of this critical group of patients were not included in the comparisons between the study groups. On the other hand, this situation made it possible to increase the homogenization of the groups and prevented the critical preoperative condition to affect the results of the study.

\section{CONCLUSION}

According to our results, the use of preoperative levosimendan infusion and the need for postoperative IABP support were higher in the patients who were admitted with ACS and had undergone CABG-only compared to the group that underwent elective CABGonly. This finding has reinforced the depressive effect of ACS on left ventricular functions. Nevertheless, there was no significant difference in mortality rates between the two groups. It may be thought that the increased need for medical practice and IABP support as well as the length of stay in the intensive care unit increase the treatment costs. However, there is no previous comparative study on this topic. Based on the results of our study and the previous studies, we believe that CABG can be safely and successfully performed in the early period with acceptable morbidity and mortality rates in ACS patients who underwent PCI for the culprit lesion and who have an indication for $C A B G$ surgery due to the remainder lesions.

\section{Declaration of authorship}

All authors have directly participated in the planning, execution, analysis or reporting of this research paper. All authors have read and approved the final version of the manuscript.

\section{Conflict of interest}

The authors disclosed no conflict of interest during the preparation or publication of this manuscript.

\section{Financing}

The authors disclosed that they did not receive any grant during conduction or writing of this study. 


\section{REFERENCES}

1. Roth GA, Huffman MD, Moran AE, Feigin V, Mensah GA, Naghavi M, et al. Global and regional patterns in cardiovasculer mortality from 1990 to 2013. Circulation 2015;132:1667-78.

2. Yalcin M, Ay D, Turk T, Yavuz S, Ozyazicioglu AF. Impact of previous percutaneous coronary intervention on postoperative outcomes of coronary artery bypass grafting. Eur Res J 2016;2:170-6.

3. Goss F, Brachmann J, Hamm CW, Haerer W, Reifart N, Levenson B. High adherence to therapy and low cardiac mortality and morbidity in patients after acute coronary syndrome systematically managed by office-based cardiologists in Germany: 1year outcomes of the ProAcor Study. Vasc Health Risk Manag 2017; 13:127-37.

4. Antman EM, Hand M, Armstrong PW, Bates ER, Green LA, Halasyamani LK, et al. 2007 focused update of the ACC/AHA 2004 guidelines for the management of patients with ST-elevation myocardial infarction: a report of the American College of Cardiology/American Heart Association Task Force on Practice Guidelines. J Am Coll Cardiol 2008;51:210-47.

5. Smith SC Jr, Dove JT, Jaccobs AK, Kennedy JW, Kereiakes D, Kern MJ, et al. ACC/AHA Guidelines for Percutaneous Coronary Intervention (Revision of the 1993 PTCA Guidelines). A Report of the American College of Cardiology/ American Heart Association Task Force on Practice Guidelines (Committee to Revise the 1993 Guidelines for Percutaneous Transluminal Coronary Angioplasty). Endorsed by the Society for Cardiac Angiography and Interventions. J Am Coll Cardiol 2001;37:2215-39.

6. Cannon CP, Weintraub WS, Demopoulos LA,Vicari R, Frey MJ, Lakkis N, et al. TACTICS (Treat Angina with Aggrastat and Determine Cost of Therapy with an Invasive or Conservative Strategy)-Thrombolysis in Myocardial Infarction 18 Investigators. Comparison of early invasive and conservative strategies in patients with unstable coronary syndromes treated with the glycoprotein IIb/IIIa inhibitor tirofiban. $\mathrm{N}$ Engl J Med 2001;344:1879-87.

7. Potluri R, Baig M, Mavi JS, Ali N, Aziz A, Uppal H, et al. The role of angioplasty in patients with acute coronary syndrome and previous coronary artery bypass grafting Int $\mathrm{J}$ Cardiol 2014; 176:760-3.

8. Yusuf S, Sleight P, Pogue J, Bosch J, Davies R, Dagenais G. Effects of an angiotensin-converting-enzyme inhibitor, ramipril, on cardiovascular events in high-risk patients. The Heart Outcomes Prevention Evaluation Study Investigators. N Engl J Med 2000;342:145-53.

9. Hoedemaker NPG, Damman P, Ottervanger JP, Dambrink JHE, Gosseling ATM, Kedhi E, et al. Trends in optimal medical therapy prescription and mortality after admission for acute coronary syndrome: a 9-year experience in a real-world setting. Eur
Heart J 2018;4:102-10.

10. Braunwald E, Domanski MJ, Fowler SE, Geller NL, Gersh BJ, Hsia J, et al. Angiotensin-converting enzyme inhibition in stable coronary artery disease. N Engl J Med 2004;351:2058-68. 11. Indio do Brasil CKO, Avezum A Jr, Uint L, Del Monaco MI, Barros VM, Campos SY, et al. Cardiovascular prevention in coronary heart disease patients: guidelines implementation in clinical practice. Rev Bras Cir Cardiovasc 2013;28:238-47.

12. Dagenais GR, Pogue J, Fox K, Simoons ML, Yusuf S. Angiotensin-converting- enzyme inhibitors in stable vascular disease without left ventricular systolic dysfunction or heart failure: a combined analysis of three trials. Lancet 2006;368:581-8. 13. Danchin N, Cucherat M, Thuillez C, Durand E, Steg PG. Angiotensin- converting enzyme inhibitors in patients with coronary artery disease and absence of heart failure or left ventricular systolic dysfunction: an overview of long-term randomized controlled trials. Arch Intern Med 2006;166:787-96.

14. Yusuf S, Pogue J. ACE inhibition in stable coronary artery disease. N Engl J Med 2005;352:937-9.

15. Lopez-Sendon J, Swedberg K, McMurray J, Tamargo J, Maggioni AP, Dargie H, et al. Expert consensus document on betaadrenergic receptor blockers. Eur Heart J 2004;25:1341-62.

16. Chen ZM, Pan HC, Chen YP,Peto R, Collins R, Jiang LX, et al. Early intravenous then oral metoprolol in 45,852 patients with acute myocardial infarction: randomised placebo-controlled trial. Lancet 2005;366:1622-32.

17. Eris C, Yavuz S, Toktas F, Turk T, Gucu A, Erdolu B, et al. Preoperative usages of levosimendan in patients undergoing coronary artery bypass grafting. Int J Clin Exp Med 2014;7:21929.

18. Lim JY, Deo SV, Rababa'h A, Altarabsheh SE, Cho YH, Hang $\mathrm{D}$, et al. Levosimendan reduces mortality in adults with left ventricular dysfunction undergoing cardiac surgery: a systematic review and meta $\square$ analysis. J Card Surg 2015;30:547-54.

19. Yavuz S, Eris C, Ata Y, Turk T. eComment. Preoperative Levosimendan Administration in Cardiac Surgery Patients. Interact Cardiovasc Thorac Surg 2013;17:714-5.

20. Jin L, Yu H, Dong T, Zhang B, Yan H, Liao H, et al. The prognostic value of ADP-induced platelet aggregation for bleeding complications in low - intermediate risk patients with acute coronary syndrome taking clopidogrel after percutaneous coronary intervention. Heart Lung Circ 2016;26:49-57.

21. Ben-Gal Y, Moses JW, Mehran R, Lansky AJ, Weisz G, Nikolsky E, et al. Surgical versus percutaneous revascularization for multivessel disease in patients with acute coronary syndromes: analysis from the ACUITY (Acute Catheterization and Urgent Intervention Triage Strategy) trial. JACC Cardiovasc Interv 2010;3:1059-67. 